\title{
Fixed-point theories on noncompact manifolds
}

\author{
Shmuel Weinberger \\ For Steve Smale, a small token of my deep admiration
}

\begin{abstract}
This paper gives three noncompact variants of Lefschetz-Nielsen fixed-point theory parallel to developments that have occurred in surgery theory. Thus, we study when a proper map can be properly homotoped, or boundedly homotoped, or even homotoped in a $C^{r}$ bounded fashion to a fixedpoint free map. As an example, the universal cover of a compact aspherical manifold always has a fixed-point free self-diffeomorphism $C^{r}$ close to the identity for all $r$ (although this is not the case, in general, for arbitrary infinite covers of such manifolds, or general universal covers).
\end{abstract}

Mathematics Subject Classification (2000).

Keywords. Fixed points, noncompact manifolds.

\section{Introduction}

By the Poincaré-Hopf theorem, a compact manifold has a nonzero vector field iff its Euler characteristic vanishes. Consequently, the vanishing of Euler characteristic is also necessary and sufficient (on a compact manifold) for the existence of a fixed-point free diffeomorphism near the identity. "Near the identity" can mean arbitrarily close in any $C^{r}$ topology. Moreover, this is compatible with the nonexistence of fixed points to be strong: the distance inf $d(x, f(x))$ can be of the same order as $d(f$, id $)$.

On the other hand, every connected noncompact manifold has a nonzero vector field. It is therefore not hard to produce diffeomorphisms close to the identity that do not have fixed points. On the other hand, we will argue that it is impossible, in general, to guarantee simultaneously that every point is moved a definite amount and for the diffeomorphism to be close to the identity.

For instance, let us assume that our manifold $M$ is given a Riemannian metric (making sense of expressions like $d(x, f(x))$ used above). We define $d_{0}(f, g)$ to be 
$\inf \left\{\sup d(f(x), g(x)), 10^{6}\right\}$. Later on we will put more structure on $M$ so as to be able to discuss norms incorporating higher derivatives of $f$ as well.

Proposition. Let $M$ be the surface of revolution of a positive curve $y=h(x)$ that is vertical at the origin and decays to 0 at infinity. Then $M$ has no diffeomorphism $f$ with $d(x, f(x))>c>0$ for all $x$.

This type of decay of an end is typical of noncompact rank 1 locally symmetric spaces (or more generally, locally symmetric spaces of $\mathbb{Q}$-rank 1 ; see [7] for a discussion). These noncompact manifolds are thus capable of admitting a theory of "almost fixed points": when they have nonzero Euler characteristic (for finite volume hyperbolic manifolds this is equivalent to their being even-dimensional), any diffeomorphism homotopic to the identity (i.e. inducing the identity on the fundamental group) must have a fixed point (using the proof of the proposition, with Lefschetz's theorem replacing Brouwer's).

Proof of Proposition. Suppose there were such an $f$. Consider a circle of rotation $C$ where the radius is less than $c / 2 \pi$. For each point $p$ on the circle, $f(p)$ cannot lie on the circle. Thus $C$ and $f(C)$ are disjoint. If $f(C)$ is "inside" $C$, then $f$ maps the 2-disk enclosed by $C$ into itself and the Brouwer theorem guarantees a fixed point. If not, consider $f^{-1}$, which maps the disk bounded by $f(C)$ into itself. A fixed point for $f^{-1}$ is also one for $f$, completing the proof.

This is perhaps not so convincing in that we use the assumption that $f$ is a diffeomorphism in our proof (on the other hand, that is an open condition in the $C^{1}$ topology). In any case, it exemplifies the main theme of this paper: it is possible to prove interesting fixed-point theorems on noncompact manifolds if one restricts the behavior of one's function at infinity. We shall discuss three different settings for such theory:

I. Our functions are required to be proper, i.e. the inverse images of compact sets are compact. This implies that the function extends to the one-point compactification of our manifold, a sometimes nice and sometimes awful space, so we will not study it by those means. (This condition is essentially opposite to the assumption of $f$ being a "compact map" oft studied in the fixed-point literature: a map is compact if its image has compact closure.)

This setting is strong enough to be able to prove some fixed-point theorems on certain finite volume locally symmetric spaces, but, interestingly, by no means on all of them. Essentially, at this level of generality, the key issues are related to Nielsen fixed-point theory and the interplay of the fundamental groups of the compact "core" of the manifold and the complements of an exhaustion.

II. A $C^{0}$ theory. Our goal is to know whether every bounded perturbation (or homotopy) of a given map is required to have a fixed point. For instance, if $M$ is compact and $f: M \rightarrow M$ is a map, then $f \times$ id: $M \times \mathbb{R} \rightarrow M \times \mathbb{R}$ is bounded distance from a fixed-point free map, but $f \times(x \mapsto-x)$ can be boundedly homotoped (i.e. homotoped so that for no point $p$ is the diameter of the path $f_{t}(p)$ 
greater than some fixed number) to a fixed-point free map only when the Lefschetz number $L(f)$ is 0 . (Indeed, the necessary and sufficient condition is that the Nielsen number $N(f)$ is 0 .)

The same is true with $\mathbb{R}^{2}$ replacing $\mathbb{R}$ and allowing any nontrivial rotation in the second coordinate.

In our discussion of this theory, we will ignore the contributions of fundamental groups that arise in case I and only discuss the new features of this setting. We shall see that there is a type of homology group associated to the map $f$ in which a Lefschetz number (i.e. class) obstructs the existence of a fixed-point free representative.

III. A theory that incorporates higher derivatives as well. In order to be able to discuss whether maps are uniformly $C^{i}$ close, we shall assume in this theory that our manifolds have bounded geometry in the following sense: the Riemannian metric on $M$ has a uniform lower bound on the injectivity radius at all points (i.e. there are no short geodesics anywhere on the manifold) and with an upper bound on all sectional curvatures and all covariant derivatives of the whole curvature tensor.

Such noncompact manifolds always have infinite volume. Nevertheless, they arise extremely naturally, e.g. as covering spaces of compact manifolds or as leaves of smooth foliations of compact manifolds. As an example, we consider Jacob's ladder, which is an infinite cyclic cover of a genus 2 surface.

Proposition. Any diffeomorphism of Jacob's ladder to itself that moves every point at least $c>0$, and is 1-close to the identity $C^{0}$, must have unbounded second derivative.

The idea, following Roe, is to consider a "normalized" Lefschetz number and to see that, if one assumes bounds on derivatives, then the number of fixed points in a ball of radius $R$ grows linearly with $R$. On the other hand, we will see the following result:

Corollary to Theorem 1. If $M$ is the universal cover of a compact aspherical manifold (i.e. $M$ is contractible and the universal cover of a compact manifold), then $M$ always admits diffeomorphisms arbitrarily close to the identity with no fixed points (or even c-almost fixed points for some $c>0$ ).

Of course, if the Euler characteristic of the manifold that $M$ covers is nonzero, then it is impossible to make such a vector field commute with all covering translates.

Our treatment here is occasionally sketchy - our goal is to explain the extent to which one can create and destroy fixed points under various kinds of deformations in the noncompact setting, distinctions that do not arise in the compact case. In some sense this paper a survey of a nonexistent literature; all of the ideas here are analogues of developments in surgery theory and/or $C^{*}$-algebra index theory, but have not been previously applied (to the best of my knowledge) in fixed-point theory. 
This paper was influenced by, at least, the work of Goresky and MacPherson on fixed sets of Hecke correspondences and in particular the role of "fixed points at infinity" [13]. This perhaps explains some of the obsession with locally symmetric spaces in the next section, the work of Heitsch and Lazarev on fixed-point theory on foliated manifolds [14] (which was, in turn, influenced by ideas of Connes and of Sullivan), and Roe [15] who liberated foliated index theorems from foliations. Finally, the paper [11] has some shared spirit with some of our proper ideas, but is rather different in its concerns.

\section{Proper maps}

Our setting for this section is a topological manifold $M$. For simplicity we will assume that $M$ has one end and is "fundamental group tame at infinity."

More precisely, we say that $M$ has one end or is connected at infinity if $M$ has an exhaustion by compact subsets $K_{1} \subset K_{2} \subset \cdots$ (whose union is $M$ and) so that $M-K_{i}$ are all connected. The "fundamental group tame" condition is that the exhaustion can be chosen so that $M-K_{i}$ all have the same fundamental group, which will be called the fundamental group at infinity.

Note that when a manifold has several cusps, each one gives a component at infinity, so there are more than one end. Also, an easy argument by interlacing exhaustions shows that the fundamental group at infinity, if defined, is well defined, and that Jacob's ladder, for instance, does not have this property. Nevertheless, it is not hard to modify the ideas of this section to handle manifolds such as these. ${ }^{1}$

See [17] for a useful discussion of the theory of ends of noncompact manifolds, and also [8] for an application of Siebenmann's ideas to the analysis of elliptic operators on manifolds and thereby to their geometry.

Now consider a proper map $f: M \rightarrow M$. We are interested in the obstruction to proper-homotoping $f$ to a fixed-point free map.

If $M$ is compact (and of dimension exceeding 2), the question of the minimum number of fixed points is completely answered by Nielsen fixed-point theory. The Nielsen element is a refinement of the Lefschetz number lying in $\mathbb{Z} \pi / \sim$, where two group elements are identified if they are $f$-conjugate (we say that $g$ and $g^{\prime}$ are $f$-conjugate if there is an $h$ in $\pi$ such that $\left.g=h g^{\prime} f(h)^{-1}\right)$. The smallest number of nonzero coefficients for the Nielsen element is the Nielsen number, and it is a (the) lower bound for the number of fixed points.

If $M$ is noncompact, then we have to identify in addition those elements of the fundamental group that differ by a curve that can be pulled outside of arbitrarily large compact sets. For instance, if $M \cong V \times \mathbb{R}^{k}$ for $k>1$ and $V$ is compact, then there is no room for any obstruction at all, as the complements of the products $V \times B^{k}$ of balls of larger and larger radii all have fundamental groups that surject

${ }^{1}$ A proper map induces an endomorphism of the ends of a manifold; in the discussion below, all that contributes to the indeterminacy in Nielsen theory is the elements coming from the (fundamental groups of the) ends that are preserved by the map. 
onto $\pi_{1}(M)$. On the other hand, if $M$ is simply connected at infinity, then the whole Nielsen element remains, except for the coefficient of the trivial element.

Sketch of proof. See the beautiful survey article [12] (and the references therein) for the classical case. In any case, the geometric definition of the Nielsen class is that one considers the (transverse) intersection of the graph $G(f)$ of $f$ with the diagonal $\Delta$ in $M \times M$ and counts intersection points according to sign and an element of fundamental group; the element of the fundamental group comes about by choosing a path to the intersection point in $G(f)$ and back to the basepoint in $\Delta$. There is an indeterminacy in each of these paths, and it is this indeterminacy that gives rise to the semiconjugacy.

When one is noncompact, there are infinitely many intersection points, i.e. fixed points of $f$, even generically. However, the points that are outside of a large compact set can only be labeled by elements of the fundamental groups of that complement. This leaves the classes that cannot be pulled to infinity with finite coefficients, and therefore an obstruction.

When the coefficients can be infinite, it is possible to deform the maps to cancel all of those coefficients: this is the old Eilenberg (Euler?) swindle $0=$ $(1-1)+(1-1)+\cdots=1+(-1+1)+(-1+1)+\cdots=1$. Concretely, one first introduces an infinite number of canceling pairs of Nielsen equivalent fixed points with opposite signs, and then follows the usual proof of cancellation of fixed points with opposite signs in the same Nielsen class from the compact case, re-pairing the collection of fixed points so that now all of the original fixed points in these homotopy classes are included.

Now let us return to the situation of a finite volume locally symmetric manifold $M$ and consider a proper self-map. These manifolds are of the form $K \backslash G / \Gamma$ where $G$ is a semisimple Lie group, $K$ is a maximal compact subgroup, and $\Gamma$ is a lattice subgroup, i.e. a subgroup for which this quotient has finite volume. If the $\mathbb{Q}$-rank of $M$ equals 1 (see [7] for how this would be defined in the nonarithmetic case) then one has the picture discussed in the introduction: for each end there is a cusp subgroup, which is a rather small subgroup of the fundamental group. Thus, there is a large group containing information about fixed points.

For instance, in the case of the punctured torus, the fundamental group is the free group $F_{2}$ and the cusp subgroup is $\mathbb{Z}$ generated by the commutator $x y x^{-1} y^{-1}$. Consequently, if one considers, for instance, a $2 \times 2$ integer matrix $A$ of determinant 1 , then $A$ induces an automorphism of the torus $\mathbb{R}^{2} / \mathbb{Z}^{2}$ fixing the origin, and hence an automorphism of the punctured torus. Straightforward calculation shows that this proper homotopy class contains $|\operatorname{det}(A-I)|-1$ fixed points.

If the $\mathbb{Q}$-rank of $M$ exceeds 1 then (as a consequence of the work of BorelSerre, Margulis, and Gromov in combination; see [7]) $M$ always has an exhaustion by compact subsets with connected complements, but whose fundamental groups always surject onto $\pi_{1}(M)$. Consequently, on these manifolds any proper self-map is homotopic to one which is fixed-point free. (Thus it is important to maintain 
much more careful control on the geometry of maps at infinity in order to obtain fixed-point theorems in this setting.)

\section{Bounded homotopy classes}

The basic question in this section is to determine when a self-map can be homotoped "boundedly," so that it is now fixed-point free. The idea is very simple, as we will see in the following case.

Suppose $f: \mathbb{R} \rightarrow \mathbb{R}$ is a nice map, say with transverse fixed points. Then we are interested in how large $|f(x)-x|$ gets on either side of each fixed point. For example, the function $f(x)=x+\sin (x)$ has infinitely many fixed points, but they can all be cancelled by a bounded homotopy $f_{t}(x)=x+t+(1-t) \sin x$ (no point moves more than 2 units during the homotopy from $t=0$ to $t=1$ ). On the other hand, the function $g(x)=x+x \sin x$ can never be homotoped to have fewer than infinitely many fixed points. Setting $x=(n+1 / 2) \pi$ gives a sequence of points for which $g(x)-x$ alternates being more and more positive and negative, and therefore any finite perturbation of $g$ will continue to have fixed points between these consecutive values of $x$.

We will follow the idea of large scale homology, or "Rips homology" $[6,16]$. Since we are dealing with 0-dimensional homology, it is not necessary to introduce the whole homology theory. If we assume that all metric balls in the manifold are simply connected, we do not need to introduce machinery to carry a Nielsentheoretic generalization. ${ }^{2}$ But, of course, for the definition of obstructions, it is not necessary to make any such assumption.

Recall the definition of locally finite homology (also known as Borel-Moore homology). It is defined like ordinary homology, using simplicial chains, except that one allows certain infinite sums; namely one can add an infinite number of simplices provided that each compact set intersects only finitely many simplices. The group $H_{0}^{l f}(X)$ is basically a product of copies of $\mathbb{Z}$, one for each compact component of $X$. For a noncompact component, all 0 -chains can be moved to infinity.

Remark. To allow nonconstant coefficient systems, they must be cosheaves, i.e. have corestriction maps (see [3]). If we use the "Nielsen ring" of the fundamental group of balls, then the 0-th locally finite homology has much less of a tendency to vanish. If one uses a proper map from a one-ended manifold to $[0, \infty)$ then the 0-th locally finite homology of the push-forward cosheaf is exactly the obstruction group arising in the previous section. One can only push to infinity classes that come from the fundamental group at infinity.

Now suppose $f: M \rightarrow M$ is a self-map. Then we have a sequence of closed subsets $F_{n}(f)=\{x \mid d(x, f(x)) \leq n\}$. Note that these inclusions are proper maps and therefore induce maps on locally finite homology. For us the relevant group

${ }^{2}$ See [20, Section 9.4A] for a discussion of the use of cosheaves to handle the issues of varying fundamental group over a "coarse space." 
is $L L(f)=\lim H_{0}^{l f}\left(F_{n}(f)\right)$. (Here $L L$ is intended to stand for "Large-scale Lefschetz.")

Proposition. There is an obstruction in $L L(f)$ to boundedly homotoping $f$ to a fixed-point free map.

The proof is quite obvious from the definitions.

Note how this proposition easily and naturally gives the phenomenon of perturbability of $f \times$ rotation: $M \rightarrow \mathbb{R}^{2} \rightarrow M \times \mathbb{R}^{2}$ to fixed-point free-ness being possible only when the rotation is trivial or $L(f)=0$. (The non-simply connected case would allow for putting in the Nielsen element in place of $L(f)$.)

Remark. In our favorite case of locally symmetric manifolds with their standard metric, if one uses the Nielsen theory, there are obstructions in all $\mathbb{Q}$-ranks, because although all elements can be pulled out to infinity, they are usually stretched to have necessarily large diameters when this is done. (In $\mathbb{Q}$-rank 1 , the diameter stays bounded during such a deformation.)

\section{Bounded geometry}

The results of this section are motivated by the results of Roe's thesis [15] and are essentially implicit in [4]. We recall that the setting is manifolds with bounded geometry: $M$ is a Riemannian manifold such that there is a uniform lower bound on the injectivity radius at all points (i.e. there are no short geodesics anywhere on the manifold) and with an upper bound on all sectional curvatures and all covariant derivatives of the whole curvature tensor. Near each point there is a coordinate ball of definite size, and the overlap maps between nearby charts have uniformly bounded partials.

The first key definition is that of amenability. A manifold of bounded geometry is amenable if there is an exhaustion by compact manifolds with boundary $K_{1} \subset K_{2} \subset \cdots$ such that $\operatorname{vol}\left(\partial K_{i}\right) / \operatorname{vol}\left(K_{i}\right) \rightarrow 0$. We call such an exhaustion regular. By a classical theorem of Følner, a finitely presented group is amenable in the sense of von Neumann (i.e. has a left invariant mean $\ell^{\infty}(\pi) \rightarrow \mathbb{R}$ ) if and only if the universal cover of some (and therefore any) compact manifold with fundamental group $\pi$ is amenable in this sense. Picking a base point in the universal cover, we can average an element of $\ell^{\infty}(\pi)$ over the translates that lie in $K_{i}$. Then we take a weak- $*$ limit of these functionals to obtain the invariant mean. Of course we can also average functions in $\ell^{\infty}(\pi)$ by the same device.

As an example, free abelian groups are amenable; regular exhaustion of Euclidean space (the universal cover of a torus) is given by balls of increasing radius.

Roe's main result was an extension of the Atiyah-Singer index theorem [2] from compact manifolds to amenable ones: he essentially shows how to define an "average index per volume" with respect to a (weak-* limit associated to a) regular exhaustion, and how to compute this in terms of a "normalized characteristic class 
formula." His typical application was to showing that, for instance, obstructions obtained using the index theorem apply as well to universal covers of obstructed manifolds in the bounded geometry setting if the fundamental group is amenable. For instance, by means of a study of the Dirac operator on a spin manifold of positive scalar curvature, he showed the following.

Theorem $([15,16])$. If $V$ is a spin manifold whose universal cover has a metric of positive scalar curvature quasi-isometric to the pullback metric, then $\int \widehat{A}(M) d \mathrm{vol}$ $=0$.

Once Roe has identified a method for generalizing [2] to appropriate operators on manifolds of bounded geometry, it is clear that one can generalize the fixedpoint theorem for elliptic complexes, which includes Lefschetz [1], to "maps of bounded geometry." Needless to say, the Lefschetz fixed-point theorem does not require the apparatus of the index theorem, and we shall not directly pursue this line of thought here.

In [5] a sort of converse to Roe's theorem on positive scalar curvature was proved: for nonamenable groups, there is always some manifold with nonvanishing integral $\int \widehat{A}(M) d$ vol whose universal cover has positive scalar curvature. In this section we will observe a similar phenomenon holds in fixed-point theory.

Corollary. A regular cover of a compact manifold $V$ has a diffeomorphism uniformly close to the identity in the $C^{2}$ topology with no c-almost fixed-points (for some $c>0$ ) if and only if either $\chi(V)=0$ or the covering group is nonamenable.

Since Cheeger and Gromov [9] have shown that all finite complexes with amenable fundamental group and contractible universal cover have zero Euler characteristic, we obtain the result that all universal covers of aspherical manifolds admit such diffeomorphisms. Obviously they can be taken to commute with the covering group iff $\chi(V)=0$.

To state the theorem of which this result is a corollary, we need to introduce some more preparation. Following [4] let us consider the complex of differential forms on a manifold of bounded geometry consisting of forms whose norms are uniformly bounded when expressed in local coefficients. (Note that in bounded geometry, this condition makes sense.) We define $H_{u f}^{i}(M)$ as the cohomology of this complex. Manifolds of bounded geometry and vector bundles with bounded curvature forms have characteristic classes that lie in these cohomology groups.

Theorem 1. If $M$ is a manifold of bounded geometry, then $M$ has a unit length vector field with bounded derivatives iff the Euler class of $M$ vanishes in $H_{u f}^{n}(M)$.

The corollary then follows from the relation between vector fields and diffeomorphisms near the identity (each point $p$ should be assigned the vector whose exponential is $f(p)$; this is possible if one has the uniform estimate $d(p, f(p))<$ inj), and the following:

Theorem 2 ([4, 5]). If $V$ is compact, with universal cover $M$, then the map induced by pulling back forms to the universal cover given by $H^{i}(V) \rightarrow H_{u f}^{i}(M)$ is 
injective in the amenable case, and is zero for the top-dimensional cohomology in the nonamenable case.

The proof of Theorem 1 is similar in spirit to the classical proof of the Poincaré-Hopf theorem. Necessity follows from the splitting of the tangent bunde that follows from such a vector field, and the definition of the Euler class in terms of Pfaffians.

Before sketching the sufficiency, it is worth understanding what is behind Theorem 2, mainly because it helps to understand how things can vanish. The injectivity statement in the amenable case holds because the averaging procedure provided by amenability applied to forms produces a splitting. For the vanishing statement in the nonamenable case, let us consider a Poincaré dual statement for 0-dimensional uniformly finite homology.

Here, imagine that $M$ is triangulated with simplices of uniformly bounded size and shape, i.e. all edges of controlled lengths, and no angles too acute. This is possible under the assumption of bounded geometry by [10]. A uniformly finite simplicial chain is a sum of simplices from this triangulation for which there is a universal bound on the coefficients of any simplex; the homology of this chain complex (with $\mathbb{Z}$ or $\mathbb{R}$ coefficients) is the uniformly finite homology. An elementary argument using the compactness of $\mathbb{R} / \mathbb{Z}$ shows the, prima facie surprising, fact that for connected noncompact manifolds, the $\mathbb{Z}$ and $\mathbb{R}$ theories agree in dimension 0 (but, obviously, not for the top dimension). We will use $\mathbb{Z}$ coefficients.

The hardest 0 -chain to make a boundary is the sum of all the vertices. (Indeed, if this element is a boundary, so is then anything [5].) Let us consider a 1-chain financially: each oriented edge describes the transfer of a dollar from initial to terminal vertex. We imagine each vertex taking part in all of the transactions demanded by a 1-chain $c$. The total change of assets at the end of all of these transactions assigns to each vertex its coefficient in $\partial c$. Thus a 1-chain whose boundary is the sum of all of the vertices would be a Ponzi scheme, i.e. a scheme of trade in which all participants gain a dollar with no one losing anything.

On $\mathbb{R}$ we know this is impossible. The amount needed to be traded goes to infinity (and some vertex defaults). The argument is easy: the amount of money that has to enter $[0, N]$ is $N$, so at least $N / 2$ crosses some endpoint, contradicting boundedness. Note how the argument invokes a regular sequence of compacta on the manifold.

On the 3-valent regular tree, it is possible. Each vertex can give up only one dollar, and receive two from neighbors. In [5] this is shown to be possible on all nonamenable manifolds; indeed, in [21], it is shown that the vertex set can be appropriately tiled by 3 -valent trees, so that this construction is general!

Now let us return to sufficiency in Theorem 1. Using the triangulation in [10] and the standard construction of vector fields on a triangulated smooth manifold (see e.g. [18]), we obtain a vector field that has zeroes forming a uniformly finite 0 -cycle representing the Poincaré dual of the Euler class. A 1-chain (with $\mathbb{Z}$ coefficients) which bounds it gives a prescription for adding canceling pairs of zeroes at 
each vertex, in such a way that they can be reassociated to now cancel each other and the original zeroes. Since the 1-chain is uniformly bounded this can be done without increasing any derivatives of the vector field too much. Then one rescales to make it unit length, completing the construction.

Remark. Using other characteristic classes, Theorem 2 leads to some normal bundles that remain nontrivial in a $C^{2}$ sense even on contractible manifolds. This has an interpretation as a coincidence formula in this setting.

\section{Acknowledgments}

I would like to thank Oliver Attie, Jonathan Block, Steve Ferry, John Roe and Kevin Whyte for many conversations related to this work. The discussion in Section 4 is essentially an elaboration of results of $[4,5]$.

Finally, it is a pleasure to dedicate this paper in honor of Steve Smale, a wise friend, who had for many years been a profound influence on me from afar, and in recent years, has been one from nearby.

This research was partially supported by NSF grants 0504721 and 0805913.

\section{References}

[1] M. Atiyah and R. Bott, A Lefschetz fixed-point formula for elliptic complexes: II, Applications. Ann. of Math. 88 (1968), 451-491.

[2] M. Atiyah and I. Singer, The index of elliptic operators, I. Ann. of Math. 87 (1968), $484-530$.

[3] G. Bredon, Sheaf Theory. 2nd ed., Grad. Texts in Math. 170, Springer, New York, 1997.

[4] O. Attie, J. Block and S. Weinberger, Characteristic classes and distortion of diffeomorphisms. J. Amer. Math. Soc. 5 (1992), 919-921.

[5] J. Block and S. Weinberger, Aperiodic tilings, positive scalar curvature and amenability of spaces. J. Amer. Math. Soc. 5 (1992), 907-918.

[6] J. Block and S. Weinberger, Large scale homology theories and geometry. In: Geometric Topology (Athens, GA, 1993), AMS/IP Stud. Adv. Math. 2.1, Amer. Math. Soc., Providence, RI, 1997, 522-569.

[7] S. Chang and S. Weinberger, Topological nonrigidity of nonuniform lattices. Comm. Pure Appl. Math. 60 (2007), 282-290.

[8] S. Chang, S. Weinberger and G. Yu, Taming three-manifolds by positive scalar curvature. Preprint, 2009.

[9] J. Cheeger and M. Gromov, $L^{2}$-cohomology and group cohomology. Topology 25 (1986), 189-215.

[10] J. Cheeger, W. Müller and R. Schrader, On the curvature of piecewise flat spaces. Comm. Math. Phys. 92 (1984), 405-454.

[11] B. Chen-Farng and D. Gottlieb, Fixed-point indices and manifolds with collars. Fixed Point Theory Appl. 2006, special issue, art. ID 87657, 8 pp.

[12] R. Geoghegan, Nielsen fixed-point theory. In: Handbook of Geometric Topology, North-Holland, Amsterdam, 2002, 499-521. 
[13] M. Goresky and R. MacPherson, Local contribution to the Lefschetz fixed-point theorem. Invent. Math. 111 (1993), 1-33.

[14] J. Heitsch and C. Lazaro, A Lefschetz theorem for foliated manifolds. Topology 29 (1990), 127-162.

[15] J. Roe, An index theorem for open manifolds, I. J. Differential Geometry 27 (1988), 87-113.

[16] J. Roe, An index theorem for open manifolds, II. J. Differential Geometry 27 (1988), $115-136$

[17] L. Siebenmann, The obstruction to putting a boundary on an open manifold. Ph.D. thesis, Princeton, 1965; http://www.maths.ed.ac.uk/ aar/surgery/sieben.pdf.

[18] M. Spivak, A Comprehensive Introduction to Differential Geometry. Vol. 1, published by M. Spivak, Brandeis Univ., Waltham, MA, 1970.

[19] C. T. C. Wall, Surgery on Compact Manifolds. London Math. Soc. Monogr. 1, Academic Press, London, 1970.

[20] S. Weinberger, The Topological Classification of Stratified Spaces. Univ. of Chicago Press, 1994.

[21] K. Whyte, Amenability, bi-Lipschitz equivalence, and the von Neumann conjecture. Duke Math. J. 99 (1999), 93-112.

[22] K. Whyte, Index theory with bounded geometry, the uniformly finite $\widehat{A}$ class, and infinite connected sums. J. Differential Geom. 59 (2001), 1-14.

Shmuel Weinberger

Department of Mathematics

University of Chicago

Chicago, IL 60637, USA

e-mail: shmuel@math.uchicago.edu

To access this journal online: www.birkhauser.ch/jfpta 